\title{
Potencial Alelopático de Duas neolignanas isoladas de folhas DE Virola surinamensis (MYRISTICACEAE)
}

\author{
Allelopathy Potential of Two Neolignans Isolated from Virola surinamensis (Myristicaceae) \\ Leaves
}

BORGES, F.C. ${ }^{2}$, SANTOS, L.S. ${ }^{3}$, CORREA, M.J.C. ${ }^{3}$, OLIVEIRA, M.N. ${ }^{2}$ e SOUZA FILHO, A.P.S. ${ }^{4}$

\begin{abstract}
RESUMO - Este trabalho teve por objetivos isolar, identificar e caracterizar a atividade alelopática de substâncias químicas presentes nas folhas de Virola surinamensis. O processo de isolamento e identificação das substâncias químicas envolveu o uso de solventes orgânicos e de Ressonância Magnética Nuclear (RMN ${ }^{1} \mathrm{H}, \mathrm{RMN}{ }^{13} \mathrm{C}$ e RMN ${ }^{13} \mathrm{C}$-DEPT), espectro de COSY e de HETCOR. A avaliação da atividade alelopática foi realizada em bioensaios de germinação de sementes, em condições de $25^{\circ} \mathrm{C}$ de temperatura constante e fotoperiodo de 12 horas, e de desenvolvimento da radícula e do hipocótilo, com $25^{\circ} \mathrm{C}$ de temperatura constante e fotoperíodo de 24 horas, empregando-se concentrações variando de 1,0 a $8,0 \mathrm{mg} \mathrm{L}^{-1}$. Como plantas receptoras, foram utilizadas as espécies daninhas Mimosa pudica, Senna obtusifolia e Senna occidentalis. Foram isoladas e identificadas duas neolignanas: a surinamensina e a virolina. A tendência geral observada nos resultados foi de aumento da intensidade dos efeitos alelopáticos inibitórios em função do aumento da concentração, com inibições máximas obtidas, sempre, na concentração de $8,0 \mathrm{mg} \mathrm{L}^{-1}$. A surinamensina apresentou maior potencial para inibir a germinação e o desenvolvimento da radícula e do hipocótilo do que a virolina, independentemente da espécie receptora e do fator da planta analisado. Considerando-se as intensidades dos efeitos promovidos sobre os três fatores das plantas, o desenvolvimento da radícula e o do hipocótilo foram mais intensamente inibidos pelas duas substâncias do que a germinação das sementes. À exceção dos efeitos verificados sobre o desenvolvimento do hipocótilo, malícia foi a espécie de maior sensibilidade aos efeitos alelopáticos das duas neolignanas, enquanto mata-pasto foi aquela que evidenciou inibições de menor magnitude.
\end{abstract}

Palavras-chave: alelopatia, inibição, planta daninha, surinamensina, virolina.

ABSTRACT - The objective of this research was to isolate, identify and characterize the allelopathic activity of chemical compounds in Virola surinamensis leaves. The process of isolation and identification of chemicals compounds involved the use of organic solvents and Magnetic Nuclear Resonance (MNR ${ }^{1} \mathrm{H}, M N{ }^{13} \mathrm{C}$ and $M N R^{13} \mathrm{C}$-DPT), HETCOR and COSY specter. Allelopathic activity was evaluated by bioassays, under constant temperature of $25^{\circ} \mathrm{C}$ and 12 - $h$ photoperiod for seed germination and $25^{\circ} \mathrm{C}$ of constant temperature and 24-hour photoperiod for radicle and hypocotyl elongation, at concentrations ranging from $1.0 \mathrm{mg} \mathrm{L}^{-1}$ to $8.0 \mathrm{mg} \mathrm{L}^{-1}$. The receiving plants used were the weeds Mimosa pudica, Senna obtusifolia and Senna occidentalis. Two neolignans were isolated and identified: surinamensin and virolin. The general trend observed was a positive relation between allelopathic effects and the concentration of the compounds, with maximum inhibition obtained at the concentration of $8.0 \mathrm{mg} \mathrm{L}^{-1}$. Surinamensin presented greater potential to inhibit seed germination and radicle/hypocotylelongation than virolin, regardless of the receiving species or plant parameter. Radicle and hypocotyl elongation were more intensely inhibited than seed germination by the two compounds. Except for the effects promoted on hypocotyl elongation, the species M. pudica was the most sensitive to the allelopathic effects caused by the two neolignans.

Keywords: allelopathy, inhibition, weed, surinamensin, virolin.

Recebido para publicação em 17.7.2006 e na forma revisada em 27.22007.

Pós-graduando em Química, Universidade Federal do Pará - UFPA, Rua Augusto Corrêa, S/N, 66075-900 Belém-PA; ${ }^{3}$ Prof. Doutor - UFPA. ${ }^{4}$ Eng.-Agr., Doutor, Embrapa Amazônia Oriental, Trav. Dr. Enéas Pinheiro, S/N, 66095-100 Belém-PA. 


\section{INTRODUÇÃO}

O desenvolvimento da atividade agrícola em áreas tropicais tem sido, em muito, limitado pelo aparecimento de uma comunidade de plantas daninhas extremamente agressivas e diversificadas, causando redução da produtividade e da lucratividade da atividade. Nas áreas de pastagens cultivadas da região Amazônica, as plantas daninhas são consideradas o principal componente dos custos de manutenção da pastagem, ao mesmo tempo em que são importantes fatores a contribuir para a redução do tempo de vida útil das pastagens.

Os atuais métodos de controles dessas plantas, especialmente o uso de herbicida sintético, não atendem mais às exigências da sociedade no tocante à preservação dos recursos naturais, da vida silvestre e do próprio homem (Anaya, 1999; Killham \& Foster, 1996). Adicionalmente, o aumento do número de espécies de plantas daninhas resistentes aos atuais herbicidas disponiveis no mercado (LeBaron, 1991; Jasieniuk et al., 1996) tem indicado que novas estratégias de controle devem ser colocadas à disposição dos agricultores, as quais, além de eficientes no controle, não se constituam em agentes fomentadores de insatisfações de ordem social.

Nesse contexto, os inúmeros metabólitos produzidos pelas plantas (Bansal \& Bhan, 1993; Langenhein, 1994) podem se constituir em fontes de novas e importantes moléculas quimicas com potencial para uso no manejo das plantas daninhas. As fitotoxinas produzidas compartilham uma característica adicional, que pode ser benéfica para o usuário e o ambiente. Como suas biossínteses são comandadas enzimaticamente, sua suscetibilidade à decomposição microbiana é geralmente alta (Cutler, 1988; Duke \& Lyndon, 1987). Logo, os produtos naturais representam, relativamente, pouco risco para o ambiente, a vida silvestre e para o próprio homem.

A região Amazônica, pela riqueza e diversidade de sua flora, pode se constituir em excelente oportunidade para prospecção de compostos químicos inovadores. Dentre os materiais disponiveis, o gênero Virola tem merecido destaque pelas suas propriedades medicinais (Barata et al., 2000). Entre as espécies desse gênero está Virola surinamensis, conhecida pela produção do comercialmente denominado "sebo de ucuúba", proveniente da gordura de suas sementes.

Estudos químicos desenvolvidos com espécies de Virola permitiram isolar compostos como alcalóides, flavonóides, lignanas, neolignanas e esteróides (Borges, 2003). Substâncias químicas pertencentes a essas classes de compostos têm sido discutidas, em diferentes trabalhos, como agentes alelopáticos (Putnam \& Tang, 1986; Whittaker \& Feeny, 1971).

Este trabalho teve por objetivos isolar e identificar substâncias químicas produzidas por Virola surinamensis e caracterizar suas atividades alelopáticas inibitórias da germinação de sementes e do desenvolvimento da radícula de plantas daninhas de áreas de pastagens cultivadas.

\section{MATERIAL E MÉTODOS}

\section{Coleta e preparo do material botânico}

A coleta do material botânico foi realizada no Campo Experimental da Embrapa Amazônia oriental, localizado em Belém, Estado do Pará. Fora coletadas apenas folhas de Virola surinamensis. As folhas colhidas foram secas em estufa de circulação forçada de ar, a temperatura de $40^{\circ} \mathrm{C}$, durante 72 horas. Em seguida, processou-se a trituração em moinho tipo Willey e acondicionou-se em sacos de plástico, em condições ambientais. $O$ resultado desse processo permitiu a obtenção de $1,5 \mathrm{~kg}$ de material seco e triturado.

\section{Procedimentos de isolamento e identificação das substâncias químicas}

O material seco e triturado foi submetido à extração exaustiva com hexano, seguido de solubilização em solução $\mathrm{MeOH} / \mathrm{H}_{2} \mathrm{O}$, na proporção de $7: 3$, e submetido à partição líquido-líquido, com os solventes hexano e clorofórmio, consecutivamente, obtendo-se a fração hexânicas, que foi fracionada por cromatografia de coluna, utilizando-se também, como adsorvente, sílica gel, e, como fase móvel, hexano/acetato de etila, com gradiente de polaridade crescente. A fração hexânica foi refracionada via CCVU, envolvendo o uso de combinação de solventes hexano/ecetato, até a obtenção de substâncias puras. 
A elucidação estrutural das substâncias químicas isoladas foi realizada, via análise de seus espectros de Ressonância Magnética Nuclear (RMN de ${ }^{1} \mathrm{H}$, RMN de ${ }^{13} \mathrm{C}$ e de experimentos de DEPT, COSY e de HETCOR).

\section{Análise da atividade alelopática das substâncias quimicas}

Bioensaios de germinação de sementes: foram desenvolvidos em câmaras, em condições de $25^{\circ} \mathrm{C}$ de temperatura constante e fotoperíodo de 12 horas. A germinação foi monitorada em período de dez dias, com contagens diárias e eliminação das sementes germinadas. Foram consideradas sementes germinadas aquelas que apresentavam extensão radicular igual ou superior a $2,0 \mathrm{~mm}$ (Duram \& Tortosa, 1985; Juntila, 1976). Cada placa de Petri de 9,0 cm de diâmetro recebeu 20 sementes, distribuídas sobre papel-filtro qualitativo.

Bioensaios de desenvolvimento da radícula e do hipocótilo: os bioensaios foram realizados em câmaras, com temperatura constante de $25^{\circ} \mathrm{C}$ e fotoperiodo de 24 horas. Cada placa de Petri de 9,0 cm de diâmetro recebeu três sementes pré-germinadas, com aproximadamente três dias de germinação. Após período de dez dias de crescimento, media-se o comprimento da radícula e do hipocótilo.

\section{Outros procedimentos experimentais}

Para avaliação dos efeitos das substâncias químicas isoladas e identificadas, utilizaram-se as concentrações de 1,$0 ; 2,0 ; 3,0 ; 4,0$ e $8,0 \mathrm{mg} \mathrm{L}^{-1}$, tendo como eluente o metanol. O volume de solução de extrato bruto e de substâncias químicas testadas foi de $3,0 \mathrm{~mL}$ por placa de Petri. A solução-teste foi adicionada apenas uma vez, quando do início de cada bioensaio, sendo, a partir de então, adicionada apenas água destilada, quando necessário. Todas as avaliações tiveram como tratamentotestemunha a água destilada.

As plantas receptoras selecionadas foram aquelas com grande ocorrência em áreas de pastagens cultivadas, que são: Mimosa pudica (malícia), Senna obtusifolia (mata-pasto) e Senna occidentalis (fedegoso). As sementes passaram por processo de limpeza e foram tratadas com ácido sulfúrico, em imersão, para quebra da dormência (Souza Filho et al., 1998).

\section{Análise estatística}

Em todos os bioensaios, o delineamento experimental foi do tipo blocos ao acaso, com três repetições. Como tratamento-testemun ha utilizou-se a água destilada. Procedeu-se à análise de variâncias pelo teste $\mathrm{F}$ e à comparação de médias pelo teste de Tukey (5\%). O programa estatístico SAS (Sas, 1989) foi utilizado para realização das análises.

\section{RESULTADOS E DISCUSSÃO}

\section{Substâncias químicas isoladas das folhas de $V$. surinamensis}

A Figura 1 apresenta, de forma sucinta, o fluxograma das diferentes fases desenvolvidas até o isolamento de duas substâncias químicas, partindo-se de $1,0 \mathrm{~kg}$ de folhas secas e moídas, seguido da extração com hexano, obtendo-se o extrato bruto hexânico. Este extrato foi submetido ao processo de eliminação de clorofila (metanol:água, na proporção de 7:3), obtendo-se uma fase hidroalcoólica. Em seguida, foi submetida à extração com hexano, fornecendo a fração hexânica. Essa fração foi refracionada em coluna de sílica eluída com hexano/acetato de etila, em polaridade crescente, originando 98 frações; da fração 48 obteve-se a mistura de duas substâncias. Na separação destas duas substâncias foi utilizada Cromatografia de Camada Delgada Preparativa com Eluição Contínua (CCDPEC).

Os dados dos espectros de RMN ${ }^{1} \mathrm{H}$ e ${ }^{13} \mathrm{C}$ da substância surinamensina são apresentados na Tabela 1 . O multipleto em $\delta 4,10(1 \mathrm{H})$ é referente ao hidrogênio $\mathrm{H}-8$. O dupleto verificado em $\delta 4,60(1 \mathrm{H}, J=8,1 \mathrm{~Hz})$ refere-se ao $\mathrm{H}-7$, acoplado com $\mathrm{H}-8$, cujo valor da constante de acoplamento $8,1 \mathrm{~Hz}$, entre $\mathrm{H}-7$ e $\mathrm{H}-8$, é indicação de que a substância pertence à série treo. Os deslocamentos químicos das $4 \mathrm{OMe}$, em $\delta 3,82, \delta 3,85, \delta 3,85$ e $\delta 3,91$, mostraram que a OMe mais desprotegida é a da posição 4, que sofre impedimento especial, ficando fora do plano do anel aromático. Na região dos hidrogênios aromáticos, $\mathrm{H}-2$ e H- 6 apresentam um único sinal em $\delta 6,60(2 \mathrm{H})$; o duplo dupleto em $\delta 6,86(1 \mathrm{H}, J=8,2$ e $2,0 \mathrm{~Hz})$ é atribuído 


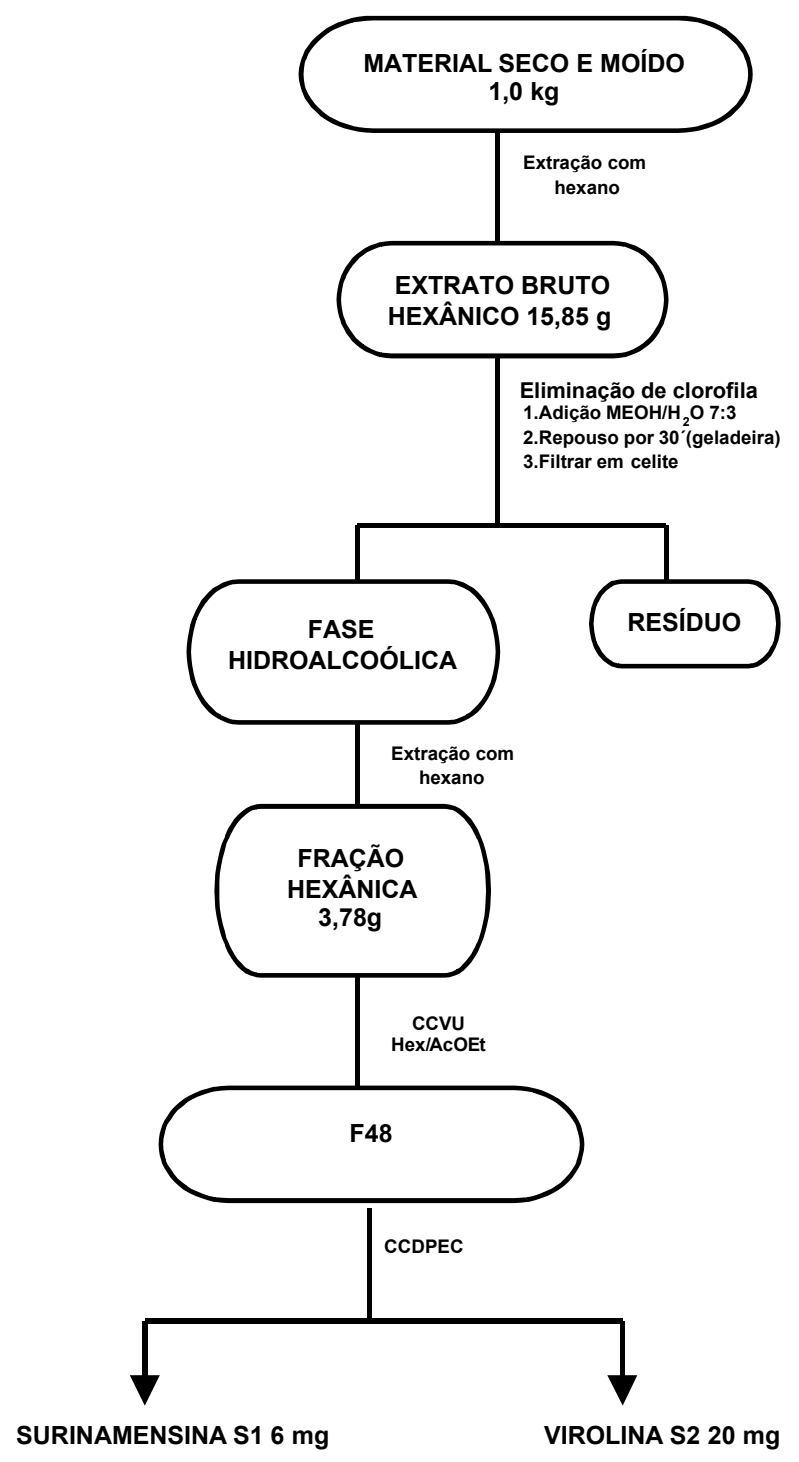

Figura 1 - Fluxograma sucinto dos procedimentos de isolamento e identificação de substâncias químicas com atividade alelopática, presentes em folhas de Virola surinamensis.

ao hidrogênio H-6'; o dupleto em $\delta$ 6,92 $(1 \mathrm{H}$, $J=2,0 \mathrm{~Hz}$ ) é referente ao $\mathrm{H}-2$ '; e o dupleto em $\delta 6,93$ é atribuído ao $\mathrm{H}-5$, que possui $J=8,2$ $\mathrm{Hz}$, resultante do acoplamento orto com H-6'.

$\mathrm{O}$ espectro de $\mathrm{RMN}{ }^{13} \mathrm{C}$ da surinamensina mostra os sinais de quatro OMe: em $\delta 55,8$, para 3'-OMe; $\delta 56,2$, atribuído a $3-\mathrm{OMe}$ e 5 -OMe, que são equivalentes; e para 4-OMe mais desprotegida, observa-se um sinal em $\delta 60,8$. O DEPT confirmou o sinal em 4-OMe em $\delta 60,8$. Os pares de carbonos $\mathrm{C}-2 / \mathrm{C}-6 \mathrm{e}$ C-3/C-5, por serem equivalentes, apresentam os sinais $\delta 104 ; 5$ e $\delta 153,3$, respectivamente. Também são observados os sinais $\delta 78,7$, $\delta 84,0$ e $\delta 17,2$, referentes aos carbonos C-7, C-8 e C-9; segundo Braga et al. (1984), esses carbonos estão mais desprotegidos que os C-7, C-8 e C-9 do isômero eritro da substância $\mathbf{S 1}$, deslocamento obtido pelo método de Beierbeck et al. (1977).

A análise conjunta desses dados e daqueles fornecidos pelos espectros de COSY e HETCOR permitiram identificar a substância $\mathbf{S 1}$ como a neolignana surinamensina (treo-1(3,4,5-tri me to xi fe ni 1) -2 - [2- me to xi-4- $(E)$ propenilfenoxi]propan-1-ol), cuja estrutura química é apresentada na Figura 2. Os dados obtidos neste trabalho estão em consonância com os de Braga et al. (1978).

Na Tabela 2 são apresentados os dados dos espectros de RMN ${ }^{1} \mathrm{H} \mathrm{e}{ }^{13} \mathrm{C}$ da substância virolina. O espectro de RMN ${ }^{1} \mathrm{H}$ dessa substância indica a presença de três grupos $\mathrm{OMe}$ em $\delta 3,87,3,88$ e 3,91 e de um duplo dupleto em $\delta 1,87(3 \mathrm{H}, J=6,5$ e $1,6 \mathrm{~Hz})$ atribuído à metila

Tabela 1 - Dados de $\mathrm{RMN}$ de ${ }^{1} \mathrm{H}\left(\mathrm{CDCl}_{3}, 300 \mathrm{MHz}\right)$ e ${ }^{13} \mathrm{C}$ $\left(\mathrm{CDCl}_{3}, 100 \mathrm{MHz}\right)$ da substância surinamensina, isolada das folhas de $V$. surinamensis

\begin{tabular}{|c|c|c|}
\hline Posição & ${ }^{1} \mathrm{H}$ & ${ }^{13} \mathrm{C}$ \\
\hline 1 & - & 147,0 \\
\hline 2 & $6,60(1 \mathrm{H}, \mathrm{s})$ & 104,5 \\
\hline 3 & - & 153,3 \\
\hline 4 & - & 135,6 \\
\hline 5 & - & 153,3 \\
\hline 6 & $6,60(1 \mathrm{H}, \mathrm{s})$ & 104,5 \\
\hline 7 & $4,60(1 \mathrm{H}, \mathrm{d}, J=8,1 \mathrm{~Hz})$ & 78,7 \\
\hline 8 & $4,10(1 \mathrm{H}, \mathrm{dq}, J=8,1 \mathrm{e} 6,2 \mathrm{~Hz})$ & 84,0 \\
\hline $9^{\prime}$ & $1,19(3 \mathrm{H}, \mathrm{d}, J=6,2 \mathrm{~Hz})$ & 17,2 \\
\hline $1^{\prime}$ & - & 133,6 \\
\hline $2^{\prime}$ & $6,92(1 \mathrm{H}, \mathrm{d}, J=2,0 \mathrm{~Hz})$ & 109,3 \\
\hline $3^{\prime}$ & - & 150,9 \\
\hline $4^{\prime}$ & - & 146,7 \\
\hline $5^{\prime}$ & $6,93(1 \mathrm{H}, \mathrm{d}, J=8,2 \mathrm{~Hz})$ & 118,8 \\
\hline $6^{\prime}$ & $6,86(1 \mathrm{H}, \mathrm{d}, J=8,2 \mathrm{e} 2,0 \mathrm{~Hz})$ & 119,2 \\
\hline $7^{\prime}$ & $6,35(1 \mathrm{H}, \mathrm{dq}, J=15,8 \mathrm{e} 1,7 \mathrm{~Hz})$ & 130,5 \\
\hline $8^{\prime}$ & $6,14(1 \mathrm{H}, \mathrm{dq}, J=15,8 \mathrm{e} 6,6 \mathrm{~Hz})$ & 124,9 \\
\hline $9^{\prime}$ & $1,87(3 \mathrm{H}, \mathrm{dd}, J=6,6 \mathrm{e} 1,7 \mathrm{~Hz})$ & 18,3 \\
\hline \multirow{3}{*}{ OMe } & $3,82(3 \mathrm{H}, \mathrm{s})$ & 55,8 \\
\cline { 2 - 3 } & $3,85(3 \mathrm{H}, \mathrm{s})$ & 56,2 \\
\cline { 2 - 3 } & $3,85(3 \mathrm{H}, \mathrm{s})$ & 56,2 \\
\hline \multirow{2}{*}{} & $3,91(3 \mathrm{H}, \mathrm{s})$ & 60,8 \\
\hline
\end{tabular}


na posição 9', que acopla com H-8' e H-7' (confirmado pelo espectro de COSY - dados não apresentados). O dupleto em $\delta 1,15(3 \mathrm{H}, J=$ $6,3 \mathrm{~Hz})$ é referente à metila na posição 9 , enquanto o dupleto centrado em $\delta \quad 4,63(1 \mathrm{H}$, $J=8,5 \mathrm{~Hz})$ e o duplo quarteto em $\delta 4,09(1 \mathrm{H}$, $J=8,5$ e $6,3 \mathrm{~Hz}$ ) são atribuídos aos hidrogênios metínicos $\mathrm{H}-7$ e $\mathrm{H}-8$, respectivamente. O valor de $J=8,5 \mathrm{~Hz}$, entre $\mathrm{H}-7$ e H-8, é uma indicação de que a neolignana $\mathbf{S 2}$ possui configuração treo (Braga et al., 1984).

Os sinais dos seis hidrogênios aromáticos aparecem entre $\delta 6,81-6,94(6 \mathrm{H}, \mathrm{m})$. O sinal duplo quarteto (distorcido) em $\delta 6,35(1 \mathrm{H})$ é referente ao hidrogênio $\mathrm{H}-7$ ', em que o valor de $J=15,7 \mathrm{~Hz}$ indica um acoplamento trans entre H-7' e H-8', em uma ligação olefinica, e o duplo quarteto em $\delta 6,14(1 \mathrm{H}, J=15,7 \mathrm{e}$ $6,5 \mathrm{~Hz}$ ) é referente ao $\mathrm{H}-8$ '.

No espectro de RMN ${ }^{13} \mathrm{C}$, podem-se observar três sinais referentes aos grupos OMe: $\delta 56,2, \delta 55,9$ e $\delta 55,8$. Os dois sinais em $\delta$ 130,5 e 124,8 são atribuídos aos carbonos olefinicos C-7' e C-8'; os sinais das metilas Me-9 e Me-9' aparecem em $\delta 17,0$ e $\delta 18,3$; e os sinais dos carbonos C-7 e C-8, em $\delta 78,4$ e 84,0 .

O espectro de COSY ${ }^{1} \mathrm{H}^{-1} \mathrm{H}$ exibe as correlações entre os hidrogênios $\mathrm{H}^{-} 7^{\prime} / \mathrm{H}-8^{\prime}$, que acoplam entre si e com a metila da posição 9'; outra correlação vista no espectro é no hidrogênio $\mathrm{H}-8$, que acopla com o hidrogênio H-7 e com os hidrogênios da posição 9 . No espectro de DEPT observa-se a presença de dez carbonos $\mathrm{CH}$.

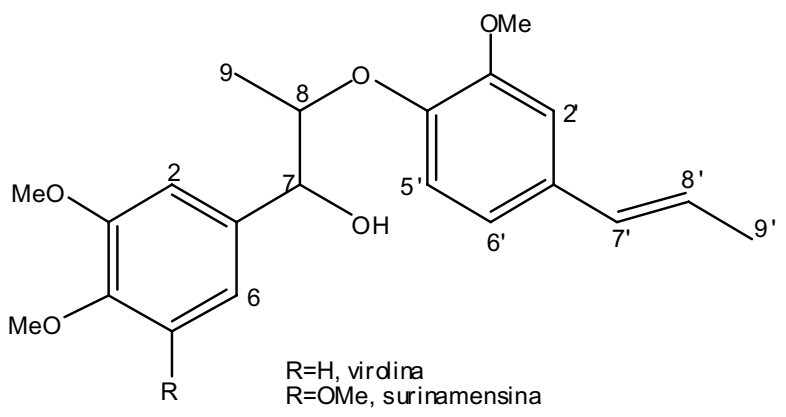

Figura 2 - Estrutura das substâncias químicas isoladas de folhas de $V$. surinamensis.
Tabela 2 - Dados de $\mathrm{RMN}$ de ${ }^{1} \mathrm{H}\left(\mathrm{CDCl}_{3}, 300 \mathrm{MHz}\right)$ e ${ }^{13} \mathrm{C}$ $\left(\mathrm{CDCl}_{3}, 100 \mathrm{MHz}\right)$ da substância virolina, isolada das folhas de $V$. surinamensis

\begin{tabular}{|c|c|c|}
\hline Posição & ${ }^{1} \mathrm{H}$ & ${ }^{13} \mathrm{C}$ \\
\hline 1 & - & 132,7 \\
\hline 2 & $6,81-6,94$ & 109,3 \\
\hline 3 & - & 148,3 \\
\hline 4 & - & 150,9 \\
\hline 5 & $6,81-6,94$ & 111,0 \\
\hline 6 & $6,81-6,94$ & 120,0 \\
\hline 7 & $4,63(1 \mathrm{H}, \mathrm{d}, \mathrm{J}=8,5 \mathrm{~Hz})$ & 78,4 \\
\hline 8 & $4,09(1 \mathrm{H}, \mathrm{dq}, \mathrm{J}=8,5 \mathrm{e} 6,3 \mathrm{~Hz})$ & 84,1 \\
\hline 9 & $1,15(3 \mathrm{H}, \mathrm{d}, \mathrm{J}=6,3 \mathrm{~Hz})$ & 17,0 \\
\hline $1^{\prime}$ & - & 133,5 \\
\hline $2^{\prime}$ & $6,81-6,94$ & 110,2 \\
\hline $3^{\prime}$ & - & 149,1 \\
\hline $4^{\prime}$ & - & 146,8 \\
\hline $5^{\prime}$ & $6,81-6,94$ & 118,8 \\
\hline $6^{\prime}$ & $6,81-6,94$ & 120,0 \\
\hline $7^{\prime}$ & $6,35(1 \mathrm{H}, \mathrm{dd}, \mathrm{J}=15,7 \mathrm{e} 1,5 \mathrm{~Hz})$ & 130,5 \\
\hline $8^{\prime}$ & $6,14(1 \mathrm{H}, \mathrm{dq}, \mathrm{J}=15,7 \mathrm{e} 6,5 \mathrm{~Hz})$ & 124,8 \\
\hline $9^{\prime}$ & $1,87(3 \mathrm{H}, \mathrm{dd}, \mathrm{J}=6,5 \mathrm{e} 1,6 \mathrm{~Hz})$ & 18,3 \\
\hline \multirow{3}{*}{ OMe } & $3,87(3 \mathrm{H}, \mathrm{s})$ & 56,2 \\
\cline { 2 - 3 } & $3,88(3 \mathrm{H}, \mathrm{s})$ & 55,9 \\
\cline { 2 - 3 } & $3,91(3 \mathrm{H}, \mathrm{s})$ & 55,8 \\
\hline
\end{tabular}

O espectro de HETCOR mostra sete correlações bem evidentes, que são: entre os sinais de carbonos dos grupos OMe em $\delta 56,2-55,8$ com os hidrogênios em $\delta 3,87-3,91, \mathrm{C}-7^{\prime}$ com H-7', C-8' com H-8', C-8 com H-8, C-7 com H-7, C-9' com H-9' e C-9 com H-9.

A análise conjunta desses dados e daqueles fornecidos pelos espectros de COSY e HETCOR permitiram confirmar a substância S2 como sendo a neolignana virolina (treo -1(3,4, dimetoxifenil)-2-[2-metoxi-4-(E)propenilfenoxi]propan-1-ol). Os dados deste trabalho estão em consonância com os de Santos et al. (1996), Bagchi et al. (1991) e Braga et al. (1984), para essa mesma substância. Na Figura 2 é apresentada a estrutura dessa substância.

\section{Avaliação da atividade alelopática das substâncias quimicas isoladas das folhas de $V$. surinamensis}

A inibição da germinação das sementes das três espécies de planta daninha variou

Planta Daninha, Viçosa-MG, v. 25, n. 1, p. 51-59, 2007 
consideravelmente, em função tanto da concentração como da substância (Tabela 3). Tanto surinamensina quanto virolina apresentaram baixo poder para inibir a germinação das sementes de mata-pasto. Malícia foi a espécie que teve a germinação das sementes inibida em maior intensidade, notadamente por surinamensina. A neolignana virolina não produziu efeitos inibitórios superiores a 4,0\% na germinação de qualquer uma das três espécies de planta daninha. As maiores reduções foram verificadas na concentração de $8,0 \mathrm{mg} \mathrm{L}^{-1}$, sendo da ordem de 31,0 e $26,0 \%$ as inibições promovidas sobre malícia e fedegoso, respectivamente. Em concentrações de até 2,0 $\mathrm{mg} \mathrm{L}^{-1}$, os efeitos não ultrapassaram o valor de $5,0 \%$.

As reduções efetivadas pela substância surinamensina estão bem próximas daquelas mostradas pelo ácido $p$-cumárico, na mesma concentração. No entanto, os resultados apresentados por virolina estão muito abaixo daqueles promovidos pelo ácido $p$-cumárico (Souza Filho et al., 2005). Considerando que as cumarinas têm sido apresentadas como potente inibidor da germinação de sementes (Rice \& Pancholy, 1973; Baskin et al., 1967), a substância surinamensina, isolada neste trabalho, aparentemente pode ser enquadrada no mesmo grupo dos potentes inibidores.

As variações nos efeitos alelopáticos efetivados pelas duas substâncias sobre o desenvolvimento da radícula foram semelhantes àquelas observadas sobre a germinação de sementes, com diferença na intensidade (Tabela 4). Os efeitos foram positivamente relacionados com a concentração, sendo as inibições máximas e mínimas observadas nas concentrações de 8,0 e 1,0 $\mathrm{mg} \mathrm{L}^{-1}$. Na espécie malícia, as inibições variaram de $29,0 \%$, na concentração de $1,0 \mathrm{mg} \mathrm{L}^{-1}$, a $77,0 \%$ na de $8,0 \mathrm{mg} \mathrm{L}^{-1}$, para a substância surinamensina. Embora em concentrações de até $3,0 \mathrm{mg} \mathrm{L}^{-1}$ as inibições no desenvolvimento da radícula de fedegoso e mata-pasto não tenham ultrapassado os $10,0 \%$, na de $8,0 \mathrm{mg} \mathrm{L}^{-1}$ elas atingiram 43,0 e 30,0\%, respectivamente, para a substância surinamensina. Para virolina, que apresentou baixo potencial para comprometer a germinação das sementes das três espécies de planta daninha, inibição da ordem de $37,0 \%$ no desenvolvimento da radícula de malícia foi obtida, embora para as espécies fedegoso e mata-pasto essa substância tenha mostrado baixo potencial como aleloquímico.

Os dados da Tabela 5 indicam que, ao contrário dos demais efeitos observados, fedegoso foi a espécie que teve o desenvolvimento do hipocótilo mais intensamente inibido pela substância surinamensina, ficando a espécie malícia como a de menor sensibilidade. Independentemente da espécie de planta daninha e da substância, os efeitos tenderam a estar positivamente relacionados à concentração, embora em algumas ocasiões essa relação não tenha sido estatisticamente diferente

Tabela 3 - Efeitos alelopáticos de duas substâncias químicas sobre a germinação de sementes de plantas daninhas. Dados expressos em percentual de inibição em relação ao tratamento-testemunha (água destilada)

\begin{tabular}{|c|c|c|c|c|}
\hline \multirow{2}{*}{ Substância } & $\begin{array}{c}\text { Concentração } \\
\left(\mathrm{mg} \mathrm{L}^{-1}\right)\end{array}$ & Malícia & Fedegoso & Mata-pasto \\
\cline { 2 - 4 } & 1,0 & $5,0 \mathrm{Da}$ & $1,0 \mathrm{Cb}$ & $1,0 \mathrm{Ab}$ \\
\hline \multirow{5}{*}{ Surinamensina } & 2,0 & $5,0 \mathrm{Da}$ & $1,0 \mathrm{Cb}$ & $1,0 \mathrm{Ab}$ \\
\cline { 2 - 4 } & 3,0 & $15,0 \mathrm{Ca}$ & $3,0 \mathrm{Cb}$ & $2,0 \mathrm{Ab}$ \\
\cline { 2 - 5 } & 4,0 & $20,0 \mathrm{Ba}$ & $16,0 \mathrm{Bb}$ & $2,0 \mathrm{Ac}$ \\
\hline \multirow{5}{*}{ Virolina } & 8,0 & $31,0 \mathrm{Aa}$ & $26,0 \mathrm{Ab}$ & $1,0 \mathrm{Ac}$ \\
& 1,0 & $2,0 \mathrm{Aa}$ & $2,0 \mathrm{Aa}$ & $1,0 \mathrm{Aa}$ \\
& 2,0 & $2,0 \mathrm{Aa}$ & $2,0 \mathrm{Aa}$ & $2,0 \mathrm{Aa}$ \\
\hline & 3,0 & $3,0 \mathrm{Aa}$ & $2,0 \mathrm{Aa}$ & $2,0 \mathrm{Aa}$ \\
\hline
\end{tabular}

Médias seguidas de letras iguais, maiúsculas na coluna e minúsculas na linha, não diferem entre si pelo teste de Tukey (5\%). 
Tabela 4 - Efeitos alelopáticos de duas substâncias químicas sobre o desenvolvimento da radícula de plantas daninhas. Dados expressos em percentual de inibição em relação ao tratamento-testemunha (água destilada)

\begin{tabular}{|c|c|c|c|c|}
\hline \multirow{2}{*}{ Substância } & $\begin{array}{c}\text { Concentração } \\
\left(\mathrm{mg} \mathrm{L}^{-1}\right)\end{array}$ & Malícia & Fedegoso & Mata-pasto \\
\cline { 2 - 5 } & 1,0 & $29,0 \mathrm{Ea}$ & $0,0 \mathrm{Dc}$ & $5,0 \mathrm{Cb}$ \\
\hline \multirow{4}{*}{ Surinamensina } & 2,0 & $34,0 \mathrm{Da}$ & $7,0 \mathrm{Cb}$ & $10,0 \mathrm{Bb}$ \\
\cline { 2 - 5 } & 3,0 & $59,0 \mathrm{Ca}$ & $7,0 \mathrm{Cb}$ & $10,0 \mathrm{Bb}$ \\
\cline { 2 - 5 } & 4,0 & $65,0 \mathrm{Ba}$ & $28,0 \mathrm{Bb}$ & $10,0 \mathrm{Bc}$ \\
\cline { 2 - 5 } & 8,0 & $77,0 \mathrm{Aa}$ & $43,0 \mathrm{Ab}$ & $30,0 \mathrm{Ac}$ \\
\hline \multirow{4}{*}{ Virolina } & 1,0 & $5,0 \mathrm{Da}$ & $4,0 \mathrm{Ba}$ & $3,0 \mathrm{Ba}$ \\
\cline { 2 - 5 } & 2,0 & $8,0 \mathrm{Da}$ & $5,0 \mathrm{ABa}$ & $5,0 \mathrm{ABa}$ \\
\cline { 2 - 5 } & 3,0 & $15,0 \mathrm{Ca}$ & $6,0 \mathrm{ABb}$ & $5,0 \mathrm{ABb}$ \\
\cline { 2 - 5 } & 4,0 & $20,0 \mathrm{Ba}$ & $6,0 \mathrm{ABb}$ & $7,0 \mathrm{ABb}$ \\
\cline { 2 - 5 } & 8,0 & $37,0 \mathrm{Aa}$ & $8,0 \mathrm{Ab}$ & $8,0 \mathrm{Ab}$ \\
\hline
\end{tabular}

Médias seguidas de letras iguais, maísculas na coluna e minúsculas na linha, não diferem entre si pelo teste de Tukey ( $5 \%$ ).

Tabela 5 - Efeitos alelopáticos de duas substâncias químicas sobre o desenvolvimento do hipocótilo de plantas daninhas. Dados expressos em percentual de inibição em relação ao tratamento-testemunha (água destilada)

\begin{tabular}{|c|c|c|c|c|}
\hline \multirow{2}{*}{ Substância } & $\begin{array}{c}\text { Concentração } \\
\left(\mathrm{mg} \mathrm{L}^{-1}\right)\end{array}$ & Malícia & Fedegoso & Mata-pasto \\
\cline { 2 - 5 } & 1,0 & $0,0 \mathrm{~Eb}$ & $0,0 \mathrm{~Eb}$ & $7,0 \mathrm{Ca}$ \\
\hline \multirow{4}{*}{ Surinamensina } & 2,0 & $3,0 \mathrm{Dc}$ & $38,0 \mathrm{Da}$ & $8,0 \mathrm{Cb}$ \\
\cline { 2 - 5 } & 3,0 & $15,0 \mathrm{Cb}$ & $48,0 \mathrm{Ca}$ & $9,0 \mathrm{Cc}$ \\
\cline { 2 - 5 } & 4,0 & $20,0 \mathrm{Bc}$ & $50,0 \mathrm{Ba}$ & $26,0 \mathrm{Bb}$ \\
\cline { 2 - 5 } & 8,0 & $27,0 \mathrm{Ac}$ & $67,0 \mathrm{Aa}$ & $35,0 \mathrm{Ab}$ \\
\hline \multirow{5}{*}{ Virolina } & 1,0 & $2,0 \mathrm{Da}$ & $2,0 \mathrm{Ca}$ & $3,0 \mathrm{Ca}$ \\
\cline { 2 - 5 } & 2,0 & $4,0 \mathrm{Da}$ & $3,0 \mathrm{Ca}$ & $5,0 \mathrm{BCa}$ \\
\cline { 2 - 5 } & 3,0 & $8,0 \mathrm{Ca}$ & $5,0 \mathrm{BCa}$ & $7,0 \mathrm{Ab}$ \\
\cline { 2 - 5 } & 4,0 & $15,0 \mathrm{Ba}$ & $7,0 \mathrm{ABb}$ & $8,0 \mathrm{Ab}$ \\
\hline
\end{tabular}

Médias seguidas de letras iguais, maiúsculas na coluna e minúsculas na linha, não diferem entre si pelo teste de Tukey (5\%).

( $p>0,05)$. Repetindo outros resultados já comentados, a virolina apresentou baixo potencial para afetar o desenvolvimento do hipocótilo das espécies fedegoso e mata-pasto, com inibições máximas, para essas duas espécies, da ordem de 9,0 e $8,0 \%$, respectivamente.

Comparando os efeitos promovidos pelas substâncias surinamensina e virolina sobre a germinação das sementes (Tabela 3) e o desenvolvimento da radícula (Tabela 4) e do hipocótilo (Tabela 5), observa-se que, independentemente da substância, o desenvolvimento da radícula, em maior extensão, e o do hipocótilo foram mais intensamente inibidos pelas substâncias do que a germinação das sementes, indicando maior sensibilidade desses parâmetros da planta aos efeitos alelopáticos das duas substâncias testadas.

Quando se observam os efeitos médios promovidos individualmente por cada substância testada sobre a germinação das sementes (Tabela 3) e o desenvolvimento da radícula (Tabela 4) e do hipocótilo (Tabela 5), verificase que, independentemente do fator da planta analisado, a surinamensina inibiu, com maior ordem de grandeza, todos os fatores analisados das plantas, indicando que essa substância possui maior potencial como aleloquímico do 
que a virolina. Os dados indicam, ainda, tendência de aumento dessa diferença com o aumento da concentração. Observando a Figura 2, verifica-se que a diferença entre as estruturas químicas das duas substâncias consiste apenas da metoxila (OMe), presente no carbono $\mathrm{C}-5$ da surinamensina, e do $\mathrm{H}$, no C-5 da virolina. Dessa forma, a maior atividade alelopática manifestada pela surinamensina pode estar associada à presença da metoxila em sua estrutura. Aparentemente, a intensidade dos efeitos alelopáticos de um dado aleloquímico estaria mais relacionada a determinadas singularidades do que ao conjunto da molécula. Souza Filho et al. (2006) apresentaram evidências a esse respeito, em trabalho de acetilação da molécula da substância titonina.

Nos últimos anos, vários trabalhos em que se analisaram a química e a atividade biológica das lignanas e neolignas foram publicados (Gottieb, 1978; Whitting, 1987; Mac Rae \& Towers, 1984). Estudos como os de Swain (1977) e Russell \& Fenemore (1973) têm classificado esses compostos como agentes de defesa contra o ataque de insetos. Chauret et al. (1996) e Gonzales-Coloma et al. (1994) destacaram o caráter inseticida das neolignanas, enquanto Zacchinos et al. (1997) demonstraram as propriedades antifúngicas delas. À semelhança dos resultados deste trabalho, Suga \& Hiraga (1998) também relataram atividade alelopática inibitória da germinação e do desenvolvimento de diferentes espécies de plantas, por neolignanas.

Neste trabalho, foram detectadas funções alelopáticas para as duas neolignanas isoladas das folhas de $V$. surinamensis, especialmente em relação à surinamensina, que apresentou grande potencial com o agente alelopático. Tal propriedade permite sua indicação como substância promissora para participar de estudos mais avançados de avaliação da atividade alelopática. Os resultados conferem, ainda, valor econômico à floresta amazônica, como fornecedora de substâncias químicas com propriedades potenciais de uso na agricultura.

\section{LITERATURA CITADA}

ANAYA, A. L. Allelopathy as a tool in the management of biotic resources in agroecosystems. Crit. Rev. Plant Sci., v. 18, n. 6 , p. 697-739, 1999.
BARATA, L. E. S. et al. Anti-leishmaniol activity of neolignans from Virola species and synthetic analogue. Phytochemistry, v. 55, n. 6, p. 580-585, 2000.

BEIERBECK, H.; SAUNDERS, J. K.; APSIMON, J. W. The semiempirical derivation of carbon-13 nuclear magnetic resonance chemical shifts. Hydrocarbons, alcohols, amines, ketones, and olefins. Can. J. Chem., v. 55, n. 15, p. 2813-2828, 1977.

BORGES, F. C. Estudo fitoquímico, alelopático e farmacológico de constituintes químicos das folhas de Virola michelii (Heckel) e Virola surinamensis (rol. Warb.). 2003. 148 f. Dissertação (Mestrado em Química) Universidade Federal do Pará, Belém, 2003.

BRAGA, A. C. H. et al. ${ }^{13} \mathrm{C}$ NMR spectral and conformational analysis of 8-O-4' neolignans. Phytochemistry, v. 23, n. 9, p. 2025-2028, 1984.

BAGCHI, A.; OSHIMA, Y.; HIKINO, H. Neolignans and lignans of Nardostachys jatamansi Roots. Planta Med., v. 57 , p. 96-97, 1991.

BANSAL, G. L.; BHAN, V. M. Status of research on allelopathy and future scop of work in Indian. Ind. J. Agric. Sci., v. 63, n. 12, p. 769-776, 1993.

BASKIN, J. M. et al. Psoralen na inhibition in the seeds of Psoralea subacaulis (Leguminosae). Phytochemistry, v. 6, n. 9, p. 1209-1213, 1967.

CHAURET, D. C. et al. Inseticidal neolignas from Piper decursens. J. Nat. Prod., v. 59, n. 2, p. 152-155, 1996.

CUTLER, H. G. Perspectives on discovery of microbial phytotoxins with herbicidal acticity. Weed Technol., v. 2, p. 525-532, 1988.

DUKE, S. O.; LYNDON, J. Herbicides from natural compounds. Weed Technol., v. 1, p. 122-128, 1987.

DURAM, J. M.; TORTOSA, M. E. The effects of mechanical and chemical scarification on germination of charlock (Sinapsis arvensis L.) seeds. Seed Sci. Technol., v. 13, n. 1, p. 155-163, 1985.

GONZALEZ-COLOMA, A. et al. Insect growth inhibitors from Machilus japonica. Phytochemistry, v. 35, n. 3, p. 607-610, 1994.

GOTTIEB, O. R. Neolignans. Fortsch. Chem. Org. Natur., v. 35, p. $1-72,1978$.

JASIENIUK, M.; BRÛLÉ-BABEL, A.; MORRINSON, I. N. The evolution and genetics of herbicide resistance in weeds. Weed Sci., v. 44, n. 1, p. 176-193, 1996. 
JUNTILA, O. Seed and embryo germination in S. vulgaris and $S$. reflexa as affected by temperature during seed development. Physiol. Plant., v. 29, p. 264-268, 1976.

KILLHAM, K.; FOSTER, R. Soil ecology. New York: Cambridge University Press, 1996. 422 p.

LANGEMHEIM, J. H. Higher plant terpenoids: a phytocentric overview of their ecological roles. J. Chem. Ecol., v. 20, n. 6, p. 1223-1282, 1994.

LeBARON, H. M. Distribution and seriusness of herbicid resistant weed infestations worldwide. In: CASELEY, J. C.; CUSSANS, G. W.; ATKIN, R. N. (Eds.). Herbicide resistances in weeds crops. Oxford: ButterworthHeinemann, 1991. p. 27-43.

MAC RAE, W. O.; TOWERS, G. N. H. Biological activities of lignans. Phytochemistry, v. 23, n. 6, p. 1207-1220, 1984.

PUTNAM, A. R.; TANG, C. S. Allelopathy: state of the science. In: PUTNAM, A. R.; TNAG C. S. (Eds.). The science of alelopathy. New York: John Wiley \& Sons, 1986. p. 1-19.

RICE, E. L.; PANCHOLY, S. K. Inhibition of nitrification by climax ecosystems. II. Additional evidence and possible role of tannins. Am. J. Bot., v. 60, n. 7, p. 691-702, 1973.

RUSSELL, G. B.; FENEMORE, P. G. New lignans from leaves of Macropiper excelsum. Phytochemistry, v. 12, n. 7, p. 1799-1803, 1973.

SANTOS, L. S. ET AL. Constituents from the leaves of Virola michelli. Fitoterapia, v. 67, n. 6, p. 555-556, 1996.
SOUZA FILHO, A. P. S.; BORGES, F. C.; SANTOS, L. S. Análise comparativa dos efeitos alelopáticos das substâncias químicas titonina e titonina acetilada. Planta Daninha, v. 24, n. 2, p. 205-210, 2006.

SOUZA FILHO, A. P. S.; PEREIRA, A. A. G.; BAYMA, J. C. Aleloquímico produzido pela gramínea forrageira Brachiaria humidicola. Planta Daninha, v. 23, n. 1, p. 25-32, 2005.

SOUZA FILHO, A. P. S.; DUTRA, S.; SILVA, M. A. M. M. Métodos de superação de dormência de sementes de plantas daninhas de pastagens cultivadas da Amazônia. Planta Daninha, v. 16, n. 1, p. 3-11, 1998.

STATISTICAL ANALYSIS SYSTEM - SAS. User's guide. Version 6.4. Cary: 1989. $846 \mathrm{p}$.

SUGA, T.; HIRAGA, Y. Allelopathic substances in ferns. Recent Res. Dev. Phytochem., v. 2, n. 2, p. 429-454., 1998.

SWAIN, T. Secondary compounds as protective agents. Ann. Rev. Plant Physiol., v. 28, p. 479-501, 1977.

ZACCHINO, S. et al. In vitro evaluation of antifungal properties oj 8'.0.4' - neolignans. J. Nat. Prod., v. 60, n. 7, p. 659-662, 1997.

WHITTAKER, R. H.; FEENY, P. P. Allelochemics: chemical interaction between species. Science, v. 171, n. 973, p. 757-770, 1971.

WHITTING O. R. Lignans, neolignans and related compounds. Nat. Prod. Reports, v. 4, p. 499-525, 1987. 